\title{
An in vivo model to assess magnesium alloys and their biological effect on human bone marrow stromal cells
}

Sayuri Yoshizawa ${ }^{1,2,3,5}$, Amy Chaya ${ }^{3,4,5}$, Kostas Verdelis $^{1,3,5}$, Elizabeth A. Bilodeau ${ }^{6}$, Charles Sfeir ${ }^{1,2,3,4,5}$

${ }^{1}$ Department of Oral Biology, ${ }^{2}$ Department of Periodontics and Preventive Dentistry, ${ }^{3}$ Center for Craniofacial Regeneration, ${ }^{4}$ Department of Bioengineering, ${ }^{5}$ McGowan Institute for Regenerative Medicine, ${ }^{6}$ Department of Diagnostic Sciences, School of Dental Medicine, University of Pittsburgh, Pittsburgh, PA, USA

Corresponding author: Charles Sfeir

Email: csfeir@pitt.edu

Phone: +1-412-648-1949

FAX: +1-412-624-6685

Office address: 598 Salk Hall

3501 Terrace Street

Pittsburgh, PA 15261

\section{Abstract}

Magnesium (Mg) alloys have many unique qualities which make them ideal candidates for bone fixation devices, including biocompatibility and degradation in vivo. Despite a rise in Mg alloy production and research, there remains no standardized system to assess their degradation or biological effect on human stem cells in vivo. In this study, we developed a novel in vivo model to assess Mg alloys for craniofacial and orthopedic applications. Our model consists of a collagen sponge seeded with human bone marrow stromal cells (hBMSCs) around a central Mg alloy rod. These scaffolds were implanted subcutaneously in mice and analyzed after eight 
weeks. Alloy degradation and biological effect were determined by microcomputed tomography (microCT), histological staining, and immunohistochemistry (IHC). MicroCT showed greater volume loss for pure Mg compared to AZ31 after eight weeks in vivo. Histological analysis showed that hBMSCs were retained around the Mg implants after 8 weeks. Furthermore, immunohistochemistry showed the expression of dentin matrix protein 1 and osteopontin around both pure Mg and AZ31 with implanted hBMSCs. In addition, histological sections showed a thin mineral layer around all degrading alloys at the alloy-tissue interface. In conclusion, our data show that degrading pure $\mathrm{Mg}$ and AZ31 implants are cytocompatible and do not inhibit the osteogenic property of hBMSCs in vivo. These results demonstrate that this model can be used to efficiently assess the biological effect of corroding Mg alloys in vivo. Importantly, this model may be modified to accommodate additional cell types and clinical applications.

\section{Key words}

Magnesium alloys, human bone marrow stromal cells, bone regeneration, microcomputed tomography, material assessment assays

\section{Introduction}

Each year, there are approximately six million bone fractures in the United States, often requiring the use of fixation devices to facilitate healing [1]. Traditionally, bone fixation devices have been made with permanent metals such as titanium alloys. Unfortunately, these materials can cause numerous long term complications and may ultimately require removal through a second surgery [2-7]. To circumvent these issues, resorbable polymers have been developed [8]; however, their lack of mechanical stability has rendered them inadequate for many load bearing applications such as mandibular and long bone fixation [9]. In addition, composite materials such as hydroxyapatite or bioglass contained polyetheretherketone (PEEK) are under development [10]; however, long term foreign body reactions to resorbable polymers have been 
reported [11-13]. For these reasons, there remains a need to develop novel materials for fracture fixation applications.

Unlike permanent metals and resorbable polymers, degradable magnesium (Mg) alloys can provide an ideal balance of degradation and strength. Mg alloys are biocompatible [14-19], have initial strength and mechanical properties similar to bone [2], and have demonstrated good osteointegration [20, 21]. Furthermore, studies have shown that $\mathrm{Mg}^{2+}$, a product of $\mathrm{Mg}$ degradation, may enhance bone formation [2, 15, 22]. For example, a recent study by Yoshizawa et al highlighted the connection between Mg ion and signaling pathways involved in bone formation, emphasizing the potential for these materials in craniofacial and orthopedic applications [23]. For these reasons, numerous $\mathrm{Mg}$ alloys are being developed; however, to our knowledge there is no method described in the literature to assess Mg alloy degradation and their cytotoxic or osteogenic behavior on human cells in vivo.

Traditionally, alloy corrosion has been studied with in vitro systems; however, in vitro corrosion is often not indicative of in vivo behavior [24-26]. Similarly, the biological effect of degrading Mg alloys is often tested in vitro through direct or indirect assays. Unfortunately, these systems are usually not suitable for studying long-term effects. Furthermore, the observed in vitro cellular response may vary significantly from in vivo tissues [14, 25]. For these reasons, we have established a new system to study Mg alloys and their biological effect in vivo.

Although this work focuses on the effect of Mg alloys on human bone marrow stromal cells (hBMSCs), this model system could be adapted for other cell types and clinical applications.

Previous studies have compared the cellular reaction of cell line (human osteosarcoma cell line MG63) and primary cultured cells (human umbilical cord perivascular cells HUCPV) to Mg alloys. Results showed a significant difference in biological response between these cell types, emphasizing that primary cells should be used for more clinically-relevant analyses [27].

Furthermore, hBMSCs have shown to form bone tissue in vivo when transplanted with certain biomaterials, such as hydroxyapatite [28], silk scaffold [29], collagen sponge [30], or even cell 
sheets without scaffold [31]. For these reasons, we identified hBMSCs as an ideal cell population to study the biological effects of Mg alloys for orthopedic and craniofacial bone regeneration.

To maintain hBMSCs around Mg alloys, a commercially available collagen sponge (Infuse ${ }^{\circledR}$ Bone Graft), without additional growth factors, was used as a scaffold (although other biocompatible scaffold could be used). Cells were pre-seeded in the sponge and transplanted with $\mathrm{Mg}$ rods into subcutaneous pockets of immunocompromised mice. We hypothesized that this model would allow us to closely study the effect of alloy degradation on cell behavior and osteogenic differentiation. Importantly, these observations allow us to make informed conclusions regarding alloy potential for craniofacial and orthopedic applications.

\section{Materials and Methods}

\subsection{Cell Culture}

Human bone marrow cells were provided to us by Dr. Albert Donnenberg (University of Pittsburgh) as previously described [32]. All procedures were approved by the University of Pittsburgh Committee on Research Involving the Dead. Cells were plated at $4 \times 10^{5} / \mathrm{cm}^{2}$ on plastic culture dishes, and colony forming cells were collected and used as hBMSCs. These cells were characterized for CD105, CD73, CD34, CD90, CD117, CD133, CD45, and CD44 expression by FACS analysis. hBMSCs were then cultured in $20 \%$ fetal bovine serum (Atlanta Biologicals, Flowery Branch, GA) contained a-minimal essential medium (a-MEM, Life Technologies, Grand Island, NY) with 1\% penicillin and streptomycin (Life Technologies, Carlsbad, CA) and 1\% L-glutamine (Life Technologies). Cells were subcultured to passage four, and $6 \times 10^{6}$ cells per sample were prepared for implantation.

\subsection{Scaffold and Alloy Preparation}


Collagen sponges (Infuse ${ }^{\circledR}$ Bone Graft, Medtronic, Minneapolis, MN) were cut into $7 \times 7 \times 3$ $\mathrm{mm}$ cubes, and a central hole for the Mg alloy was punched using a $3 \mathrm{~mm}$ diameter Dermapunch $^{\mathrm{TM}}$ (Figure 1). The collagen sponges were soaked in culture medium, blotted (dried) with filter paper, and immediately transferred to cell containing medium at $37^{\circ} \mathrm{C}$ for 30 minutes to facilitate cell adhesion. Two types of Mg alloys were assessed in this study, $99.9 \%$ pure $\mathrm{Mg}$ and $\mathrm{AZ31}$ (contains 3\% aluminum and 1\% zinc; Goodfellow, Coraopolis, PA). Alloys were machined as $3 \times 3 \mathrm{~mm}$ cylinders, heat treated at $205^{\circ} \mathrm{C}$ for 1.5 hours, and furnace cooled. Alloys were then washed by sonication in pure acetone and ethanol for 10 minutes each, and sterilized under UV light for two hours on all sides. All alloys were kept sterile until implantation. Mg alloys without cell seeded collagen sponges were similarly prepared and used as controls.

\subsection{Surgical Procedure}

All protocols for animal experiments were approved by the Institutional Animal Care and Use Committees at the University of Pittsburgh. Eight to ten week old immunocompromised mice (BALB/C nude) were purchased from Charles River Laboratories International (Wilmington, MA). A $2 \mathrm{~cm}$ central dorsal incision was made and subcutaneous pockets were created by blunt dissection. Samples were transplanted into individual pockets and incisions were closed with surgical clips. Samples were harvested after eight weeks, dissected with surrounded tissue, and formalin fixed for subsequent analysis.

\subsection{Microcomputed Tomography}

Mg alloys were scanned by microcomputed tomography (microCT; VivaCT 40, Scanco Medical AG, Brüttisellen, Switzerland) before implantation and after dissection. Scans were performed at $55 \mathrm{kV}, 72 \mu \mathrm{A}$, with an integration time of $139 \mathrm{~ms}$ (10.5 $\mu \mathrm{m}$ isotropic voxel). After scanning, alloys were segmented from background and soft tissue using adaptive thresholding as previously described $[33,34]$. Thresholds were defined for each sample based on distinct 
density differences between the Mg alloy and surrounding soft tissue. VivaCT 40 software was then used to analyze alloy morphology and quantify volume.

\subsection{Histological Processing}

Dissected samples were embedded in Technovit 9100 New $^{\circledR}$ (Heraeus Kulzer, Hanau, Germany), allowing sectioning of alloys and mineralized tissue without decalcification. Samples were sectioned at $6 \mu \mathrm{m}$ and stained with Hematoxylin and Eosin, von Kossa, and Alizarin Red. hBMSCs were identified by in situ hybridization using human specific Alu probe with In Situ Hybridization and Detection Kit (Rembrandt, Amsterdam, Netherland) according to manufacturer's instruction. Bone protein expression was analyzed by immunohistochemistry (IHC) using primary antibodies against human and mouse dentin matrix protein 1 (DMP1; SC6551, Santacruz, Santa Cruz, CA) and human and mouse osteopontin (OPN; SC-10593, Santacruz) with IgG Alexa Fluor ${ }^{\circledR} 594$ secondary antibody (Life Technologies, Carlsbad, CA). Bright field and fluorescent images were obtained using a Nikon Eclipse TE2000-E microscope (Nikon Instruments, Melville, NY). The inflammation in the surrounding tissue of transplants was analyzed by a pathologist.

\subsection{Statistics}

Statistical analysis was performed using IBM SPSS Statistics 19 (IBM, Armonk, NY). Alloy volume loss was compared between material groups ( $n=4$ per group) using a Student's t-test. Graphical representations reflect mean \pm standard deviation.

\section{Results}

\subsection{Pure Mg lost significantly more volume than AZ31 after eight weeks in vivo}


Four samples of each alloy were scanned by microCT before implantation and after eight weeks to quantify volume changes due to corrosion in vivo. MicroCT 3D reconstructions showed that both pure Mg and AZ31 underwent pitting corrosion; however, pure Mg samples showed larger voids ( $\leq 0.2 \mathrm{~mm}$ diameter) (Figure 2A). Alloy volume quantification showed significantly greater volume loss for pure Mg compared to AZ31 after eight weeks in vivo (Figure 2B). Pure Mg degradation yielded a net decrease in volume of $1.4 \mathrm{~mm}^{3}$, while AZ31 yielded a net decrease of $0.5 \mathrm{~mm}^{3}$.

\section{2. hBMSCs, calcium and phosphate precipitate, and an appropriate immune response were} observed around all alloys

Mg alloys and surrounding tissue were embedded in Technovit $9100 \mathrm{New}^{\circledR}$. Blocks were sectioned perpendicular to the long axis of the Mg rod for visualization of the alloy-tissue interface. Most $\mathrm{Mg}$ alloy sections were not retained during the sectioning or staining processes; however, their original location remained clearly visible and has been indicated with asterisks throughout. Hematoxylin and Eosin staining showed normal tissue morphology and cellularity around all Mg alloys (Figure 3 A-D). An appropriate inflammatory response was observed for all samples, including fibrous capsule formation (Supplemental Figure 1). For $99.9 \% \mathrm{Mg}$, a mixture, but predominately low intensity inflammatory infiltrate comprised of predominately lymphocytes, plasma cells, macrophages was seen. For AZ31, a low to moderate intensity inflammatory infiltrate comprised of a mixture of predominately lymphocytes, plasma cells, and macrophages was seen (Supplemental Figure 1). No multinucleated giant cells were seen in sections analyzed. hBMSCs were identified by in situ hybridization using human specific Alu probe, and were observed surrounding and often directly in contact with the Mg alloys (Figure 3 E-F). hBMSCs appeared flat and formed multiple layers around the alloys. Mineral formation was analyzed by von Kossa and Alizarin Red. In all samples, a thin (5-20 $\mu \mathrm{m})$ layer containing calcium and phosphate was present at the alloy-tissue interface (Figure 4). In some samples, 
this mineral layer appears separated from surrounding tissue due to sectioning artifact caused by the separation of the $\mathrm{Mg}$ alloy during sectioning.

\subsection{Bone marker proteins were expressed surrounding degrading alloys}

Osteogenic protein expression was analyzed by immunohistochemistry (IHC). For alloys implanted with hBMSCs, DMP1 (Figure 5) and OPN (Figure 6) expression was observed a short distance $(\sim 100 \mu \mathrm{m})$ from the alloy-tissue interface. In contrast, for alloys implanted without hBMSCs, no bone marker protein expression was observed. Although no quantitative analysis was performed, the AZ31 group appeared to have slightly higher expression of DMP1 and OPN.

\section{Discussion}

Due to their unique combination of biocompatibility, degradation, and mechanical strength, Mg alloys are being studied for numerous craniofacial and orthopedic applications. As an increasing number of alloys are created, there is a growing need to develop a model to assess the biological effect of alloy degradation on human cells in a cost efficient rodent model. Importantly, studies have shown that many traditional in vitro assays cannot accurately predict in vivo behavior [24-26], suggesting that an in vivo assessment model is necessary.

In the current study, we used commercially available $99.9 \%$ pure $\mathrm{Mg}$ and AZ31 to elucidate appropriate analysis methods for Mg alloys. AZ31 contains 3\% aluminum and $1 \%$ zinc, and was originally produced to improve corrosion resistance for industrial applications. However, its corrosion resistance and biocompatibility suggests its promise as a candidate biomaterial for bone fixation [16]. Although, excess amounts of aluminum have shown to be toxic and associated with neurological disorders [35], recent in vivo studies using sheep suggest that the amount of aluminum released by degrading $A Z 31$ bone fixation screws is within the safe range [36]. For this reason, we identified AZ31 as an appropriate slow degrading alloy to compare with faster degrading pure $\mathrm{Mg}$. 
To begin assessing the implanted alloys, we assessed in vivo corrosion behavior. Currently, several methods are used to study alloy corrosion in vitro and in vivo, including weight measurements [25, 37]. This method is largely destructive, requiring surrounding tissue and corrosion products to be chemically removed, therefore inhibiting the ability to study corrosion product production or the alloy-tissue interface. For these reasons, we chose to use high resolution microCT to study changes in alloy volume $[25,33]$. To do so, net alloy volume change was observed visually and quantified. Our data show that $99.9 \%$ pure $\mathrm{Mg}$ rods underwent significantly greater volume loss than AZ31 after eight weeks in vivo. Interestingly, it was previously reported that the corrosion rate of $99.99 \%$ pure $\mathrm{Mg}$ and AZ31 are similar in a rat subcutaneous implant model (99.99\% pure Mg: $0.221 \mathrm{~mm} / \mathrm{year}$, and AZ31: $0.223 \mathrm{~mm} /$ year after 21 days) [38]. This suggests a significant difference in the degradation behavior of $99.9 \%$ and 99.99\% pure Mg; with $99.9 \% \mathrm{Mg}$ degrading faster than highly pure $99.99 \% \mathrm{Mg}$ in vivo. This difference in corrosion rate is likely attributed to microgalvanic corrosion acceleration caused by impurities in the $99.9 \%$ pure material [39].

Both pure $\mathrm{Mg}$ and $\mathrm{Mg}$ alloys are prone to localized pitting corrosion [25, 39]. During degradation, a thin corrosion resistant layer forms on the alloy surface; however this layer is easily disrupted due to Mg's reactivity with chloride in the body. Disruption of this layer allows corrosion to perpetuate, causing surface particles to break off. This localized corrosion spreads across the alloy surface and may eventually cover the entire alloy [39]. This type of corrosion changes the shape of alloys in vivo, and subsequently affects their mechanical properties. This corrosion behavior, along with hydrogen-assisted cracking and transgranular cracking could ultimately cause $\mathrm{Mg}$ alloy fracture [40]. For these reasons, understanding alloy corrosion behavior is crucial while studying Mg alloys for load-bearing devices. Preference should be given to alloys which display corrosion behavior most similar to surface corrosion, thereby avoiding premature catastrophic device failure. 
Another key characteristic of degrading alloys is their biological effect. Traditionally, the biological effect of Mg alloys has been studied in vitro by seeding cells directly on Mg alloys [14, 16 , 17]. However, cells often behave differently in vivo due to protein deposition, long term material exposure, and changes in extracellular matrix [24]. In addition, the degradation rates of $\mathrm{Mg}$ alloys vary in vitro and in vivo. For these reasons, in vivo models are often preferred, such as calvarial or femoral defects [41] [42]. These functional models allow assessments of biological responses such as bone regeneration and osseointegration; however, they are typically limited to accommodating a small number of implants per animal, and do not allow us to analyze effects on clinically-relevant human cells.

In contrast, our subcutaneous model described herein utilizes a commercially available collagen sponge seeded with human bone marrow stromal cells and a central Mg rod. Collagen sponges have been used for bone regeneration for decades due to their excellent biocompatibility, cell infiltration capacity, and degradation property [45-47]. In this study, the collagen sponge served as an ideal carrier to maintain cells around degrading alloys. We recognize that this model cannot fully replicate a functional model (such as those previously described) due to variations in local tissue environment and subsequent Mg alloy corrosion rate; however, it provides many advantages to traditional tissue culture methods. In addition, it utilizes a relatively inexpensive lower rodent model while facilitating clinically-relevant assessments of the effect on human cells.

Since Mg alloys exhibit potential as bone fixation devices, we have chosen to study the effect of their degradation on hBMSCs. Specifically, we have focused on assessing mineralization and osteogenic differentiation of cells surrounding the alloys. In situ hybridization and histological sections showed that hBMSCs and host cells remained around the degrading implants, demonstrating the efficacy of this model as a relatively low cost approach to assessing hBMSCs in vivo. 
Around the alloys, detailed histologic analysis revealed a low to moderate local inflammatory response, as has been similarly shown with other degradable Mg alloys [19], including fibrous capsule formation. In addition, von Kossa and Alizarin Red staining identified a calcium and phosphate rich layer surrounding all samples. This observation is consistent with several other reports of mineral formation around degrading Mg alloys [15-18, 48]. In the present study, this mineral layer was observed around all alloys, suggesting there was no effect of the implanted hBMSCs on this mineral layer formation. Interestingly however, we did observe differences in bone marker protein expression based on the presence of hBMSCs. IHC of established bone marker proteins DMP1 and OPN showed expression surrounding degrading alloys implanted with hBMSCs. Since no bone protein expression was observed in samples without hBMSCs, we speculate that these proteins are either secreted from hBMSCs or hBMSCs influenced secretion of these proteins from host mouse cells. We are currently pursuing quantitative analysis of these proteins to provide additional detail. Interestingly, protein expression was observed approximately $100 \mu \mathrm{m}$ away from the Mg implants. This expression pattern could suggest that as the $\mathrm{Mg}$ alloys degraded, a gradient of $\mathrm{Mg}$ ion was diffused into surrounding tissue. Based on our previous in vitro study, we hypothesize that this gradient stimulated the cells' osteogenic activity when it reached a certain, optimal concentration [23]. These findings might also explain the higher expression of proteins around AZ31 (slower corroding) compared to pure Mg (faster corroding).

\section{Conclusion}

In this study, we have developed and tested a novel model to study the biological effect $\mathrm{Mg}$ alloys on human cells in a mouse model. We have demonstrated the effectiveness of our model in assessing Mg alloys based on their degradation behavior and subsequent biological effect on both host tissue and implanted hBMSCs in vivo. These data will allow us to more accurately 
identify $\mathrm{Mg}$ alloys which are well suited for bone fixation devices. Uniquely, this model may also be tailored to analyze the biological effect of $\mathrm{Mg}$ release from alloys on various cell types.

\section{Acknowledgments}

This study was supported by NSF ERC for Revolutionizing Metallic Biomaterials (grant 0812348). The authors thank Andrew Holmes (University of Pittsburgh) for Mg alloy machining, Da-Tren Chou and Dr. Prashant N. Kumta (University of Pittsburgh) for Mg alloy heat treatment, Dr. Frank Witte (Berlin, Germany) for histological guidance, and Dr. Arnold Donnenberg (University of Pittsburgh) for providing hBMSCs.

\section{Disclosures}

The authors have no conflicts of interest to disclose. 


\section{Figure Legends}

Figure 1. Scaffold schematic of Mg alloy rod with surrounding collagen sponge seeded with hBMSCs Schematic shows degradation of central Mg rod over time, causing Mg ion release into surrounding scaffold and cell population.

Figure 2. Pure Mg lost significantly more volume than AZ31 at 8 weeks MicroCT 3D renderings and volume quantification shows significantly greater volume loss for pure $\mathrm{Mg}$ compared to $\mathrm{AZ31}$ rods in vivo. MicroCT reconstructions show corrosion of pure $\mathrm{Mg}$ and $\mathrm{AZ31}$ rods after 8 weeks $(A)$. Net volume loss was quantified from reconstructed volumes before implantation and after 8 weeks in vivo (B). Averages presented as mean values \pm standard deviations. A student's t-test was used to determine significant differences ( ${ }^{*} p<0.05$ ) between material groups. Volume loss of pure Mg rods was significantly greater than AZ31 rods. Scale bars are $1 \mathrm{~mm}$.

Figure 3. Transplanted hBMSCs were detected around Mg alloys Hematoxylin and Eosin stained sections of pure Mg and AZ31 with hBMSCs (A \& B) and without hBMSCs (C \& D) show cell localization around implants. In situ hybridization with human specific Alu probe on pure Mg $(E)$ and $A Z 31(F)$ identifies human cells within the surrounding cell population. hBMSCs were observed directly surrounding Mg alloys, within fibrous tissue. * indicates implant location. Scale bars are $100 \mu \mathrm{m}$.

Figure 4. Mineral layer was formed at the alloy-tissue interface Alizarin Red staining of pure $\mathrm{Mg}$ and $\mathrm{AZ31}$ with hBMSCs (A \& B) and without hBMSCs (C \& D), and von Kossa staining of pure Mg and AZ31 with hBMSCs (E \& F) and without hBMSCs ( $G \& H$ ) show the presence of a calcium and phosphate rich layer at the alloy-tissue interface. * indicates implant location. Scale bars are $100 \mu \mathrm{m}$.

Figure 5. DMP1 was expressed surrounding Mg alloys Immunofluorescent staining for DMP1. DMP1 expression was observed a short distance from the alloy-tissue interface for pure 
Mg and AZ31 samples with hBMSCs. DMP1 expression was not observed around alloys implanted without hBMSCs. * indicates implant location. Scale bars are $100 \mu \mathrm{m}$.

Figure 6. OPN is expressed surrounding Mg alloys Immunofluorescent staining for OPN. OPN expression was observed a short distance from the alloy-tissue interface for pure $\mathrm{Mg}$ and AZ31 samples with hBMSCs. OPN expression was not observed around alloys implanted without hBMSCs. * indicates implant location. Scale bars are $100 \mu \mathrm{m}$.

\section{Supplemental Figure 1. Appropriate immune response was observed around Mg alloys} Hematoxylin and Eosin staining of tissue surrounding pure $M g(A)$ and $A Z 31(B)$. Mild chronic inflammation was observed around pure $\mathrm{Mg}$, and mild to moderate chronic inflammation was observed around AZ31. Arrows indicate inflammatory cells (L: lymphocyte, P: plasma cell, and M: mast cell). * indicates implant location. Scale bars are $50 \mu \mathrm{m}$. 


\section{References}

[1] Bergen GS, National Center for Health Statistics (U.S.). Injury in the United States : 2007 chartbook. Hyattsville, MD: U.S. Department of Health and Human Services, Centers for Disease Control and Prevention, National Center for Health Statistics; 2008.

[2] Staiger MP, Pietak AM, Huadmai J, Dias G. Magnesium and its alloys as orthopedic biomaterials: A review. Biomaterials 2006;27:1728-34.

[3] Hanson B, van der Werken C, Stengel D. Surgeons' beliefs and perceptions about removal of orthopaedic implants. BMC musculoskeletal disorders 2008;9:73.

[4] Busam ML, Esther RJ, Obremskey WT. Hardware removal: indications and expectations. The Journal of the American Academy of Orthopaedic Surgeons 2006;14:113-20.

[5] Pietak A, Mahoney P, Dias GJ, Staiger MP. Bone-like matrix formation on magnesium and magnesium alloys. Journal of materials science Materials in medicine 2008;19:407-15.

[6] Puleo DA, Huh WW. Acute toxicity of metal ions in cultures of osteogenic cells derived from bone marrow stromal cells. Journal of applied biomaterials : an official journal of the Society for Biomaterials 1995;6:109-16.

[7] Jacobs JJ, Gilbert JL, Urban RM. Corrosion of metal orthopaedic implants. The Journal of bone and joint surgery American volume 1998;80:268-82.

[8] Mantripragada VP, Lecka-Czernik B, Ebraheim NA, Jayasuriya AC. An overview of recent advances in designing orthopedic and craniofacial implants. J Biomed Mater Res A 2013;101:3349-64.

[9] Claes L, Ignatius A. Development of new, biodegradable implants. Chirurg 2002;73:9906.

[10] Abdullah MR, Goharian A, Abdul Kadir MR, Wahit MU. Biomechanical and bioactivity concepts of polyetheretherketone composites for use in orthopedic implants-a review. J Biomed Mater Res A 2015.

[11] Bostman 0, Pihlajamaki H. Clinical biocompatibility of biodegradable orthopaedic implants for internal fixation: a review. Biomaterials 2000;21:2615-21.

[12] Bostman OM, Pihlajamaki HK. Adverse tissue reactions to bioabsorbable fixation devices. Clinical Orthopaedics and Related Research 2000:216-27.

[13] Fraser RK, Cole WG. Osteolysis after Biodegradable Pin Fixation of Fractures in Children. Journal of Bone and Joint Surgery-British Volume 1992;74:929-30.

[14] Gu XN, Zheng YF, Cheng Y, Zhong SP, Xi TF. In vitro corrosion and biocompatibility of binary magnesium alloys. Biomaterials 2009;30:484-98.

[15] Witte F, Kaese V, Haferkamp H, Switzer E, Meyer-Lindenberg A, Wirth CJ, et al. In vivo corrosion of four magnesium alloys and the associated bone response. Biomaterials 2005;26:3557-63.

[16] Chou DT, Hong D, Saha P, Ferrero J, Lee B, Tan Z, et al. In vitro and in vivo corrosion, cytocompatibility and mechanical properties of biodegradable Mg-Y-Ca-Zr alloys as implant materials. Acta Biomater 2013;9:8518-33. 
[17] Hong D, Saha P, Chou DT, Lee B, Collins BE, Tan Z, et al. In vitro degradation and cytotoxicity response of Mg- $4 \% \mathrm{Zn}-0.5 \% \mathrm{Zr}$ (ZK40) alloy as a potential biodegradable material. Acta Biomater 2013;9:8534-47.

[18] Willbold E, Kalla K, Bartsch I, Bobe K, Brauneis M, Remennik S, et al. Biocompatibility of rapidly solidified magnesium alloy RS66 as a temporary biodegradable metal. Acta Biomater 2013;9:8509-17.

[19] Witte F, Ulrich H, Rudert M, Willbold E. Biodegradable magnesium scaffolds: Part 1: appropriate inflammatory response. J Biomed Mater Res A 2007;81:748-56.

[20] Castellani C, Lindtner RA, Hausbrandt P, Tschegg E, Stanzl-Tschegg SE, Zanoni G, et al. Bone-implant interface strength and osseointegration: Biodegradable magnesium alloy versus standard titanium control. Acta Biomaterialia 2011;7:432-40.

[21] Witte F, Ulrich H, Palm C, Willbold E. Biodegradable magnesium scaffolds: Part II: periimplant bone remodeling. J Biomed Mater Res A 2007;81:757-65.

[22] Janning C, Willbold E, Vogt C, Nellesen J, Meyer-Lindenberg A, Windhagen H, et al. Magnesium hydroxide temporarily enhancing osteoblast activity and decreasing the osteoclast number in peri-implant bone remodelling. Acta Biomaterialia 2010;6:1861-8. [23] Yoshizawa S, Brown A, Barchowsky A, Sfeir C. Magnesium ion stimulation of bone marrow stromal cells enhances osteogenic activity, simulating the effect of magnesium alloy degradation. Acta Biomater 2014;10:2834-42.

[24] Habibovic P, Woodfield T, de Groot K, van Blitterswijk C. Predictive value of in vitro and in vivo assays in bone and cartilage repair - What do they really tell us about the clinical performance? Tissue Engineering 2006;585:327-60.

[25] Walker J, Shadanbaz S, Woodfield TB, Staiger MP, Dias GJ. Magnesium biomaterials for orthopedic application: a review from a biological perspective. Journal of biomedical materials research Part B, Applied biomaterials 2014;102:1316-31.

[26] Witte F, Fischer J, Nellesen J, Crostack HA, Kaese V, Pisch A, et al. In vitro and in vivo corrosion measurements of magnesium alloys. Biomaterials 2006;27:1013-8.

[27] Feyerabend F, Fischer J, Holtz J, Witte F, Willumeit R, Drucker H, et al. Evaluation of short-term effects of rare earth and other elements used in magnesium alloys on primary cells and cell lines. Acta Biomaterialia 2010;6:1834-42.

[28] Mankani MH, Kuznetsov SA, Fowler B, Kingman A, Robey PG. In vivo bone formation by human bone marrow stromal cells: Effect of carrier particle size and shape. Biotechnol Bioeng 2001;72:96-107.

[29] Meinel L, Fajardo R, Hofmann S, Langer R, Chen J, Snyder B, et al. Silk implants for the healing of critical size bone defects. Bone 2005;37:688-98.

[30] Mueller SM, Glowacki J. Age-related decline in the osteogenic potential of human bone marrow cells cultured in three-dimensional collagen sponges. J Cell Biochem 2001;82:58390.

[31] Akahane M, Nakamura A, Ohgushi H, Shigematsu H, Dohi Y, Takakura Y. Osteogenic matrix sheet-cell transplantation using osteoblastic cell sheet resulted in bone formation without scaffold at an ectopic site. J Tissue Eng Regen Med 2008;2:196-201.

[32] Donnenberg AD, Gorantla VS, Schneeberger S, Moore LR, Brandacher G, Stanczak HM, et al. Clinical implementation of a procedure to prepare bone marrow cells from cadaveric vertebral bodies. Regenerative medicine 2011;6:701-6.

[33] Henderson SE, Verdelis K, Maiti S, Pal S, Chung WL, Chou DT, et al. Magnesium alloys as a biomaterial for degradable craniofacial screws. Acta Biomater 2014;10:2323-32. 
[34] Chaya A, Yoshizawa S, Verdelis K, Noorani S, Costello BJ, Sfeir C. Fracture healing using degradable magnesium fixation plates and screws. J Oral Maxillofac Surg 2015;73:295-305. [35] Macdonald TL, Martin RB. Aluminum ion in biological systems. Trends Biochem Sci 1988;13:15-9.

[36] Willbold E, Kaya AA, Kaya RA, Beckmann F, Witte F. Corrosion of magnesium alloy AZ31 screws is dependent on the implantation site. Materials Science and Engineering BAdvanced Functional Solid-State Materials 2011;176:1835-40.

[37] Witte F, Fischer J, Nellesen J, Vogt C, Vogt J, Donath T, et al. In vivo corrosion and corrosion protection of magnesium alloy LAE442. Acta Biomaterialia 2010;6:1792-9. [38] Walker J, Shadanbaz S, Kirkland NT, Stace E, Woodfield T, Staiger MP, et al. Magnesium alloys: Predicting in vivo corrosion with in vitro immersion testing. J Biomed Mater Res B 2012;100B:1134-41.

[39] Song GL, Atrens A. Understanding magnesium corrosion - A framework for improved alloy performance. Advanced Engineering Materials 2003;5:837-58.

[40] Choudhary L, Raman RKS. Magnesium alloys as body implants: Fracture mechanism under dynamic and static loadings in a physiological environment. Acta Biomaterialia 2012;8:916-23.

[41] Tu Q, Valverde P, Li S, Zhang J, Yang P, Chen J. Osterix overexpression in mesenchymal stem cells stimulates healing of critical-sized defects in murine calvarial bone. Tissue Eng 2007;13:2431-40.

[42] Xing J, Jin H, Hou T, Chang Z, Luo F, Wang P, et al. Establishment of a bilateral femoral large segmental bone defect mouse model potentially applicable to basic research in bone tissue engineering. The Journal of surgical research 2014;192:454-63.

[43] Song GL, Atrens A. Corrosion mechanisms of magnesium alloys. Advanced Engineering Materials 1999;1:11-33.

[44] Witte F, Hort N, Vogt C, Cohen S, Kainer KU, Willumeit R, et al. Degradable biomaterials based on magnesium corrosion. Current Opinion in Solid State \& Materials Science 2008;12:63-72.

[45] Geiger M, Li RH, Friess W. Collagen sponges for bone regeneration with rhBMP-2. Advanced Drug Delivery Reviews 2003;55:1613-29.

[46] Friess W. Collagen - biomaterial for drug delivery. European Journal of Pharmaceutics and Biopharmaceutics 1998;45:113-36.

[47] Chvapil M. Collagen Sponge - Theory and Practice of Medical Applications. J Biomed Mater Res 1977;11:721-41.

[48] Bowen PK, Drelich J, Goldman J. Magnesium in the murine artery: probing the products of corrosion. Acta Biomater 2014;10:1475-83. 


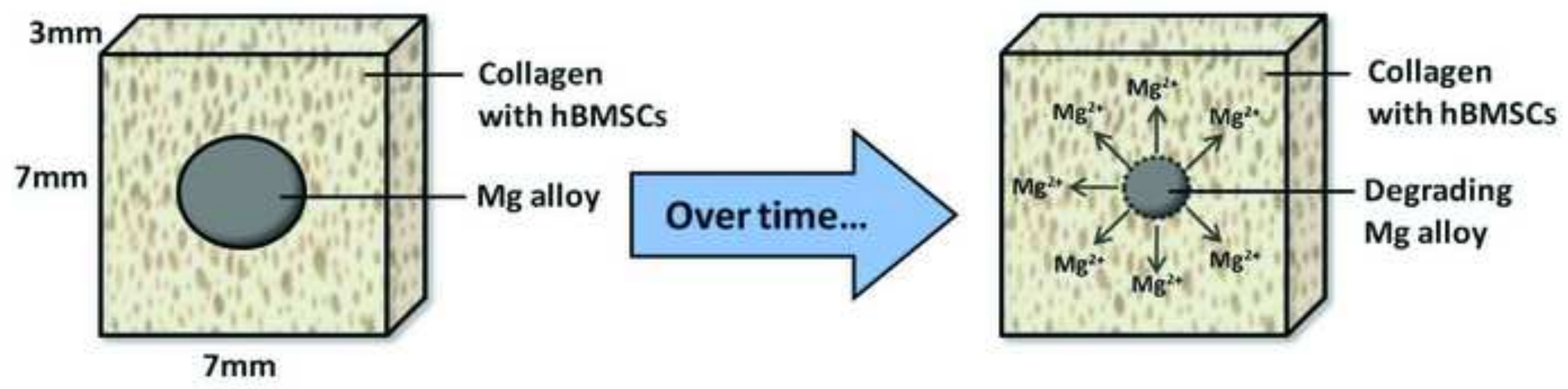



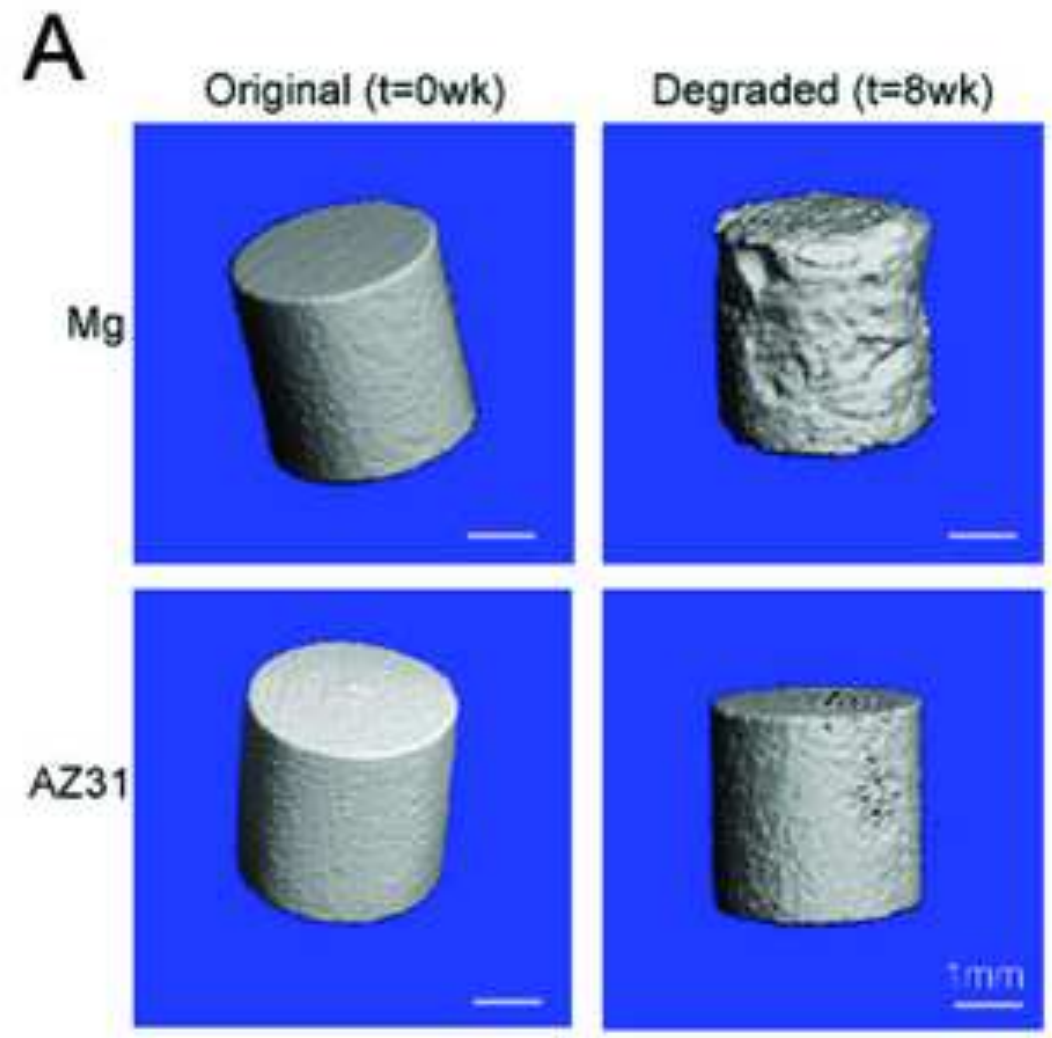

B

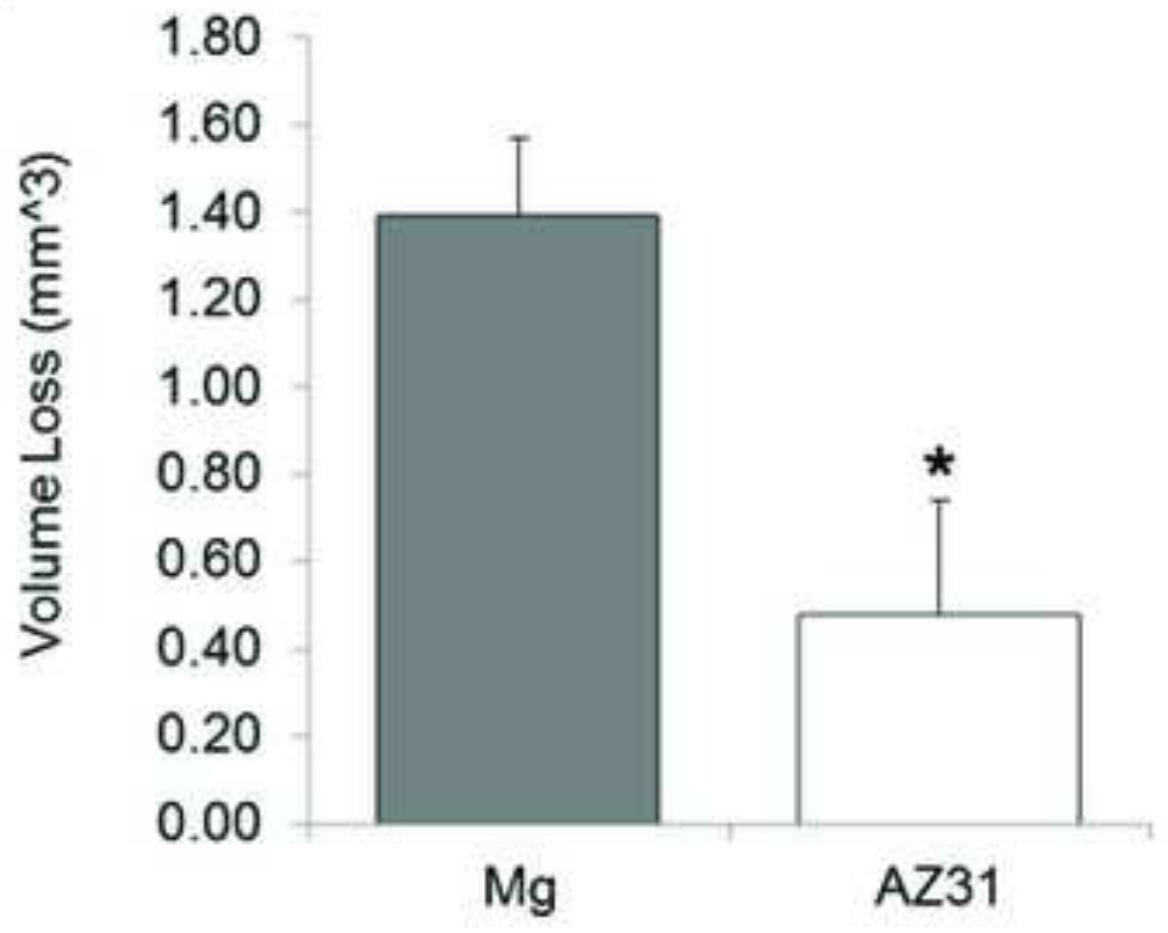


Pure Mg

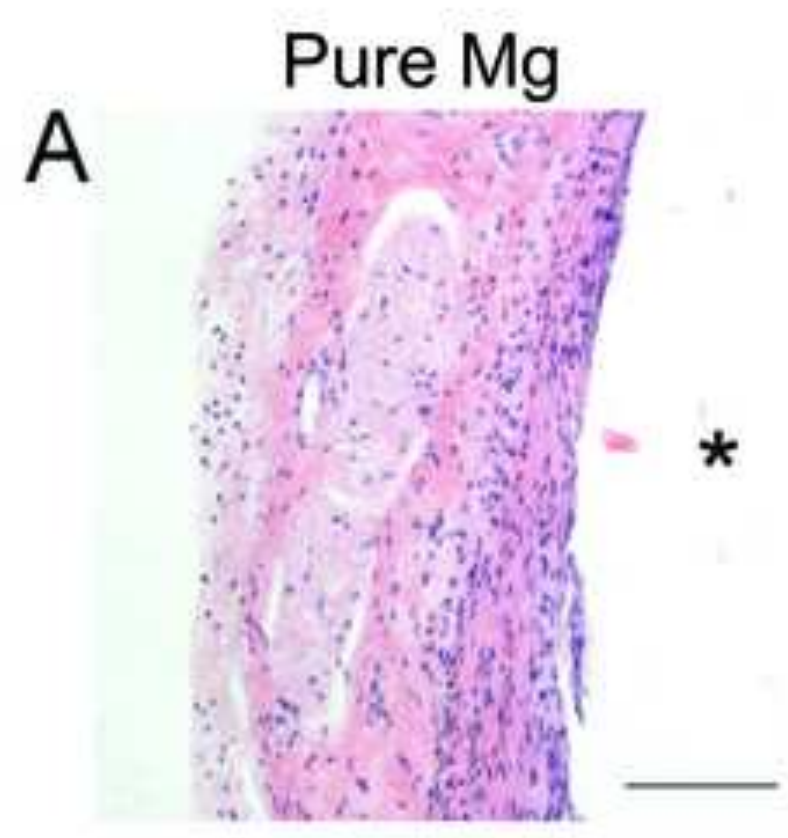

C

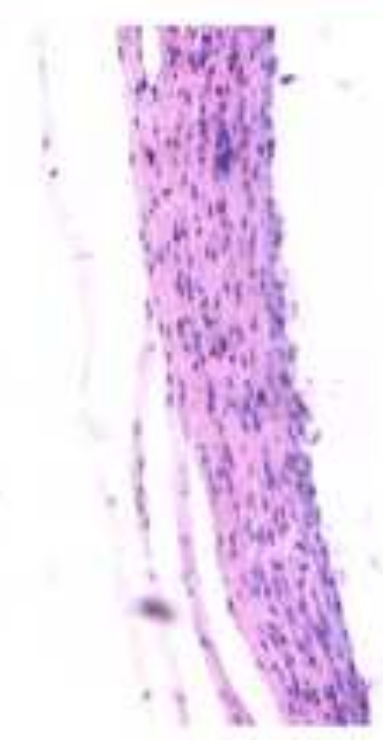

*

E
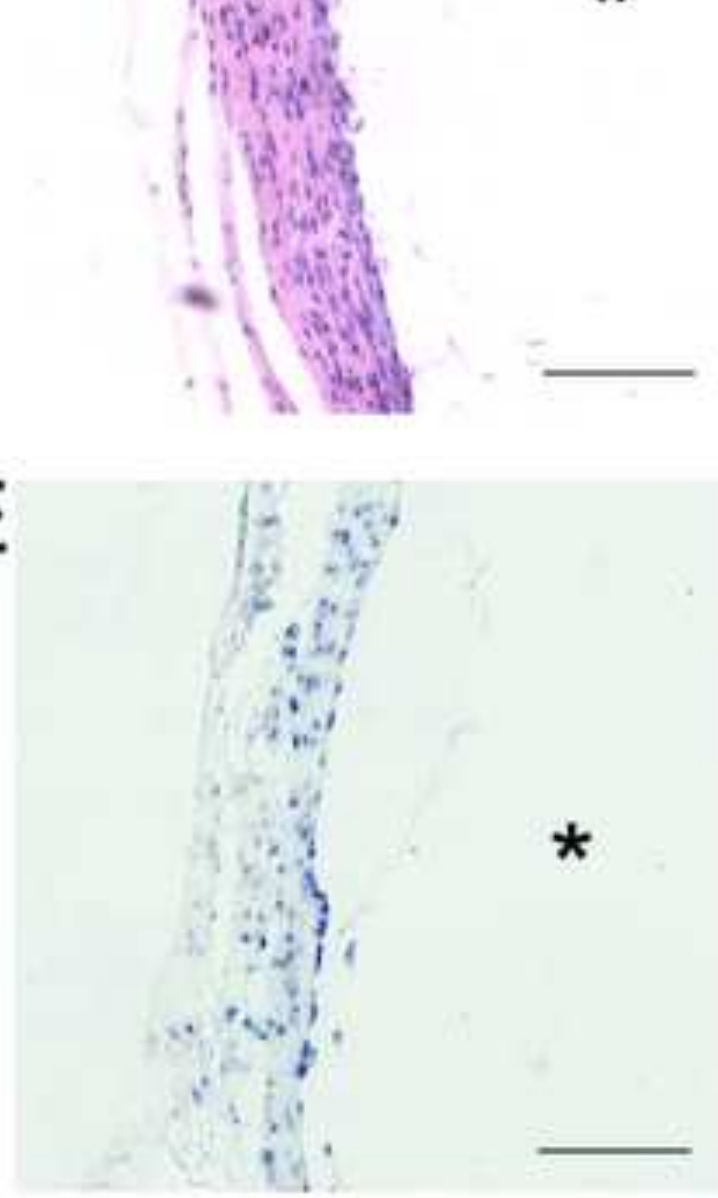
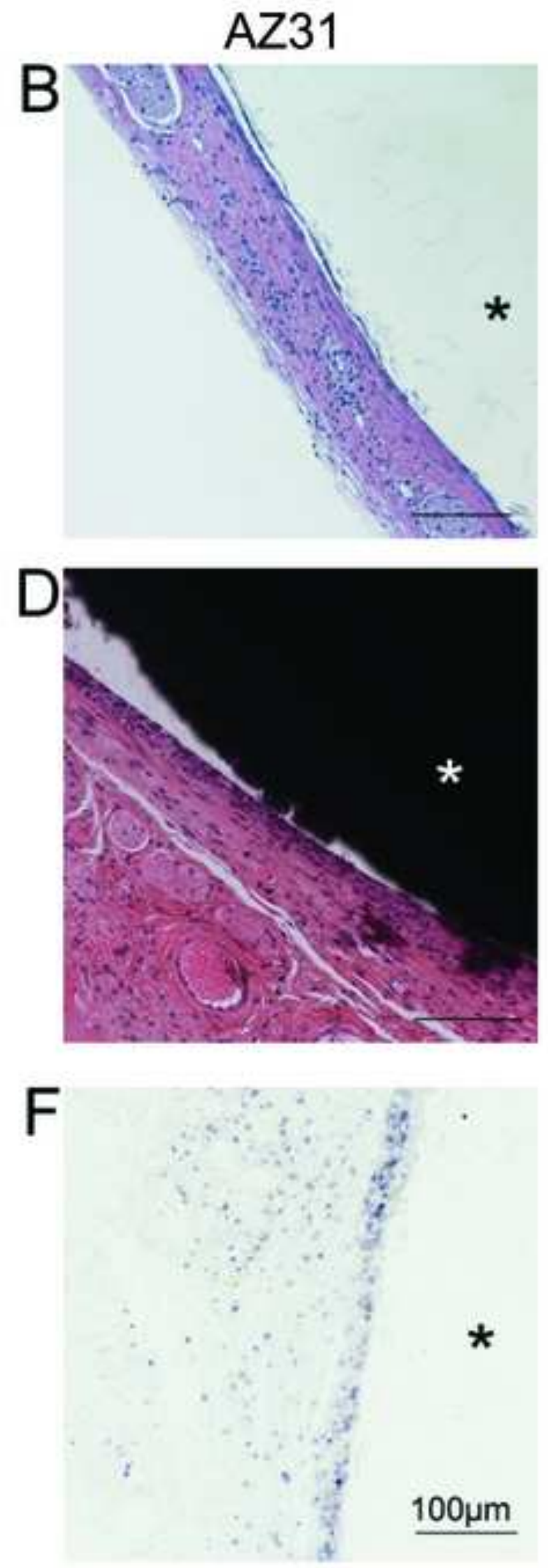

Figure 3 
With hBMSCs
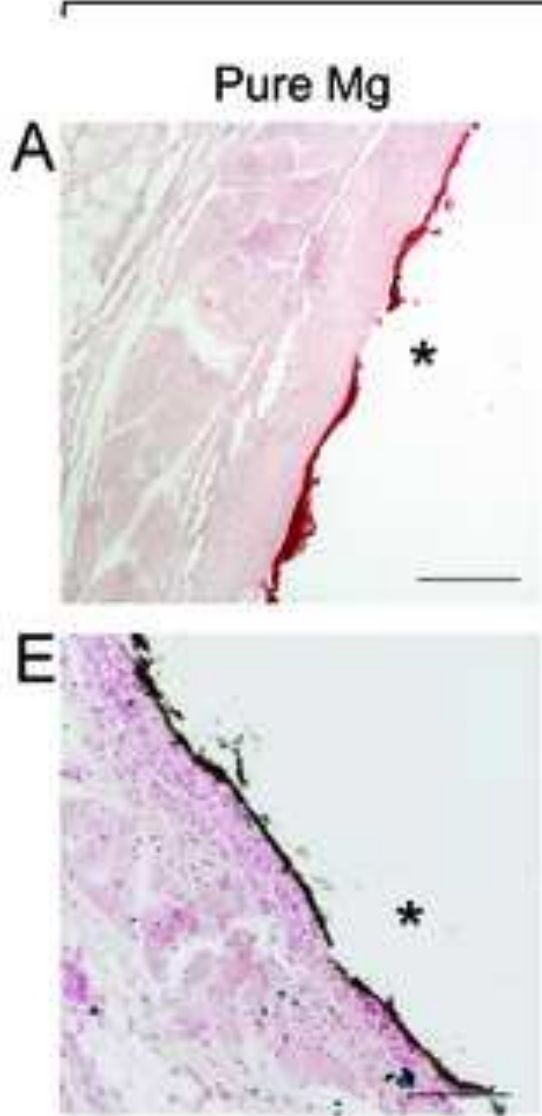

AZ31

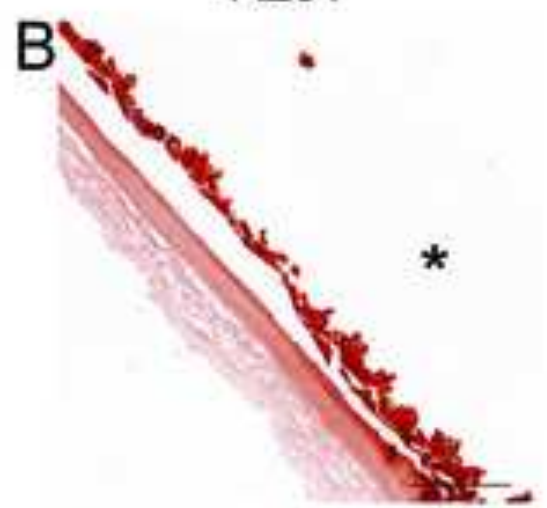

F

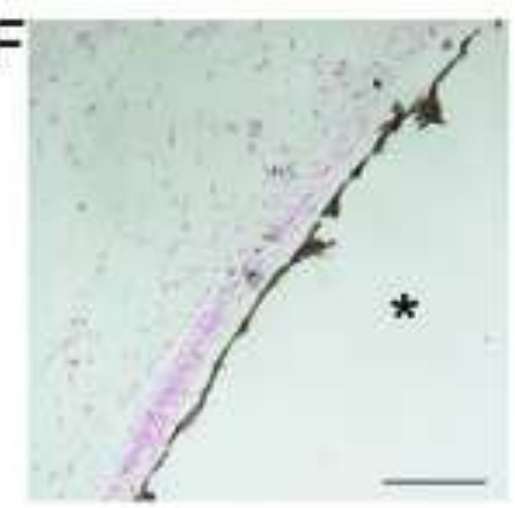

Without hBMSCs

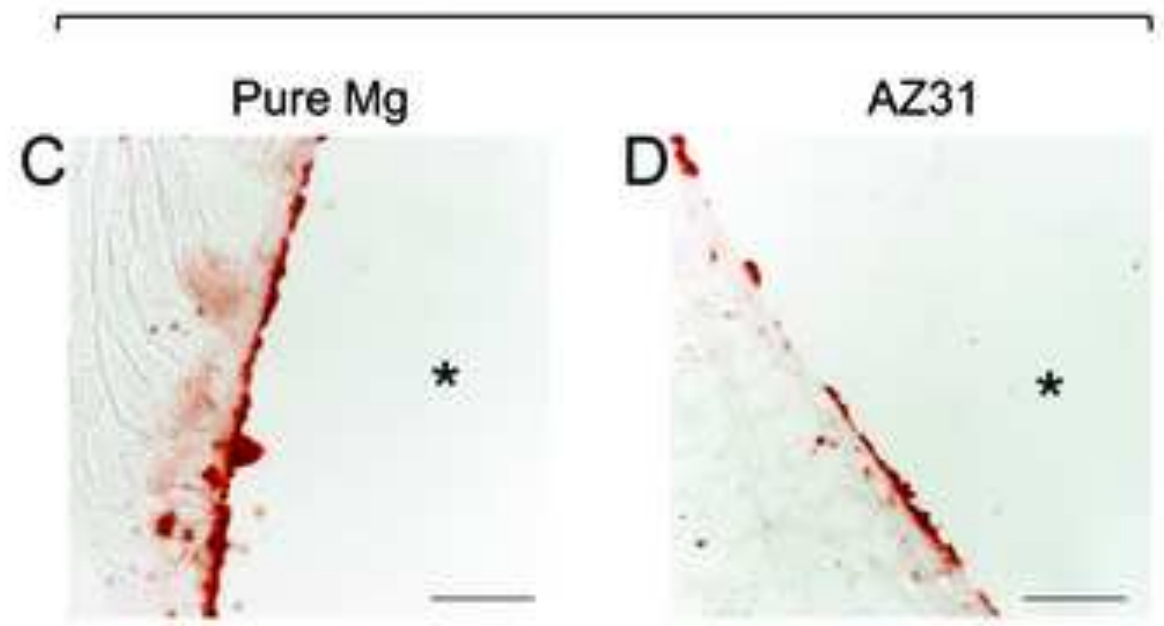

G
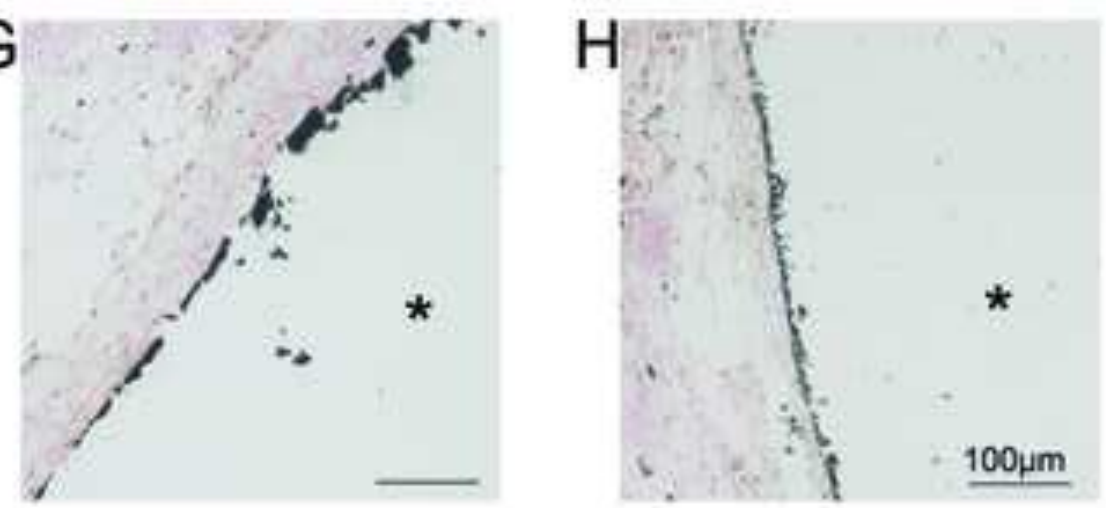


\section{Pure Mg}

With hBMSCs

Without hBMSCs
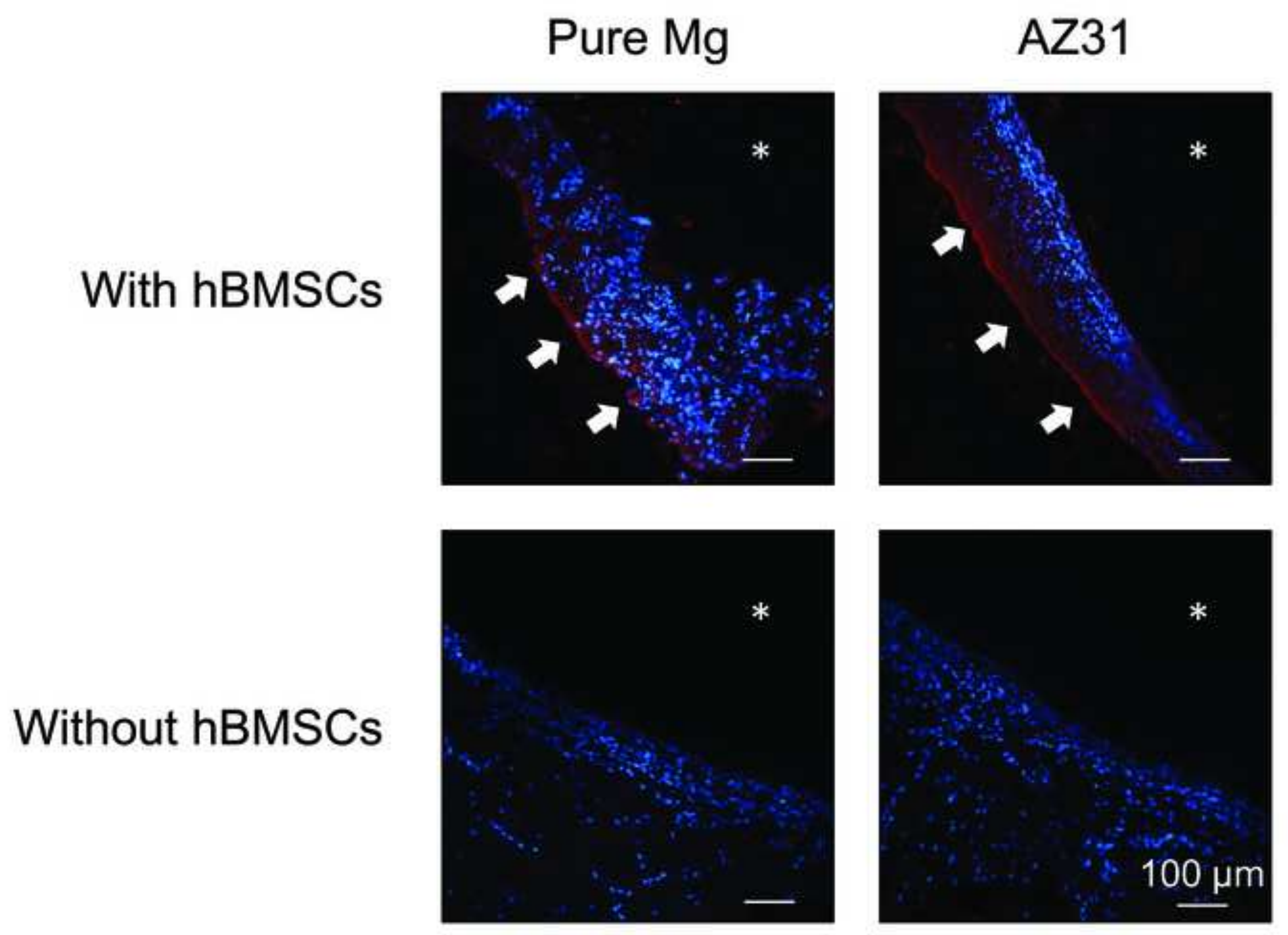

AZ31

*
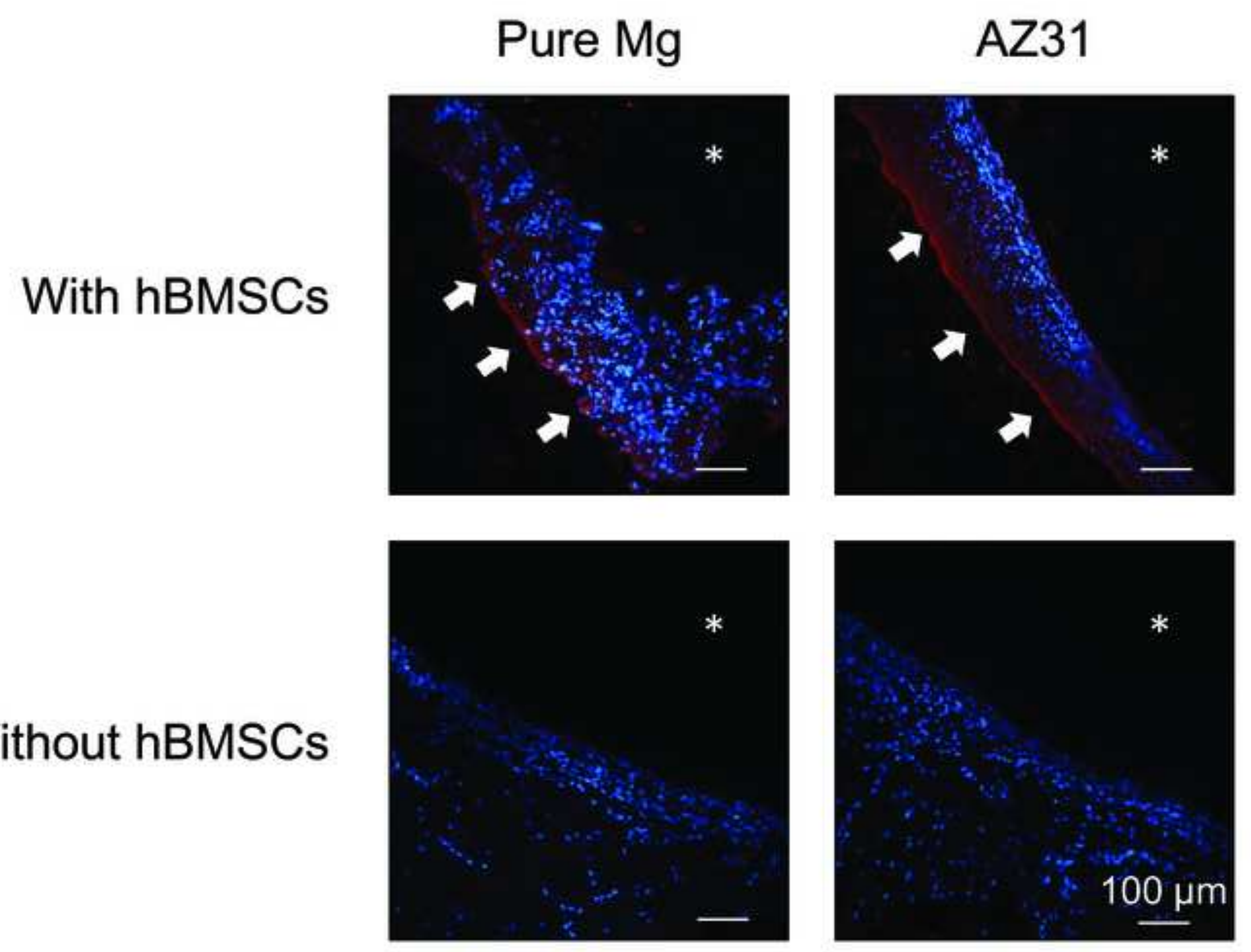
Pure Mg

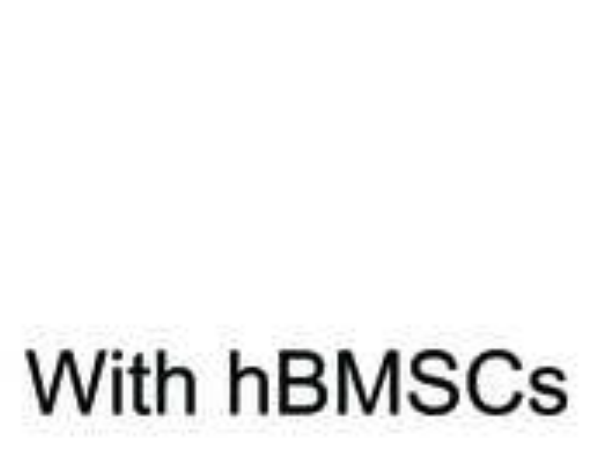

Without hBMSCs
AZ31
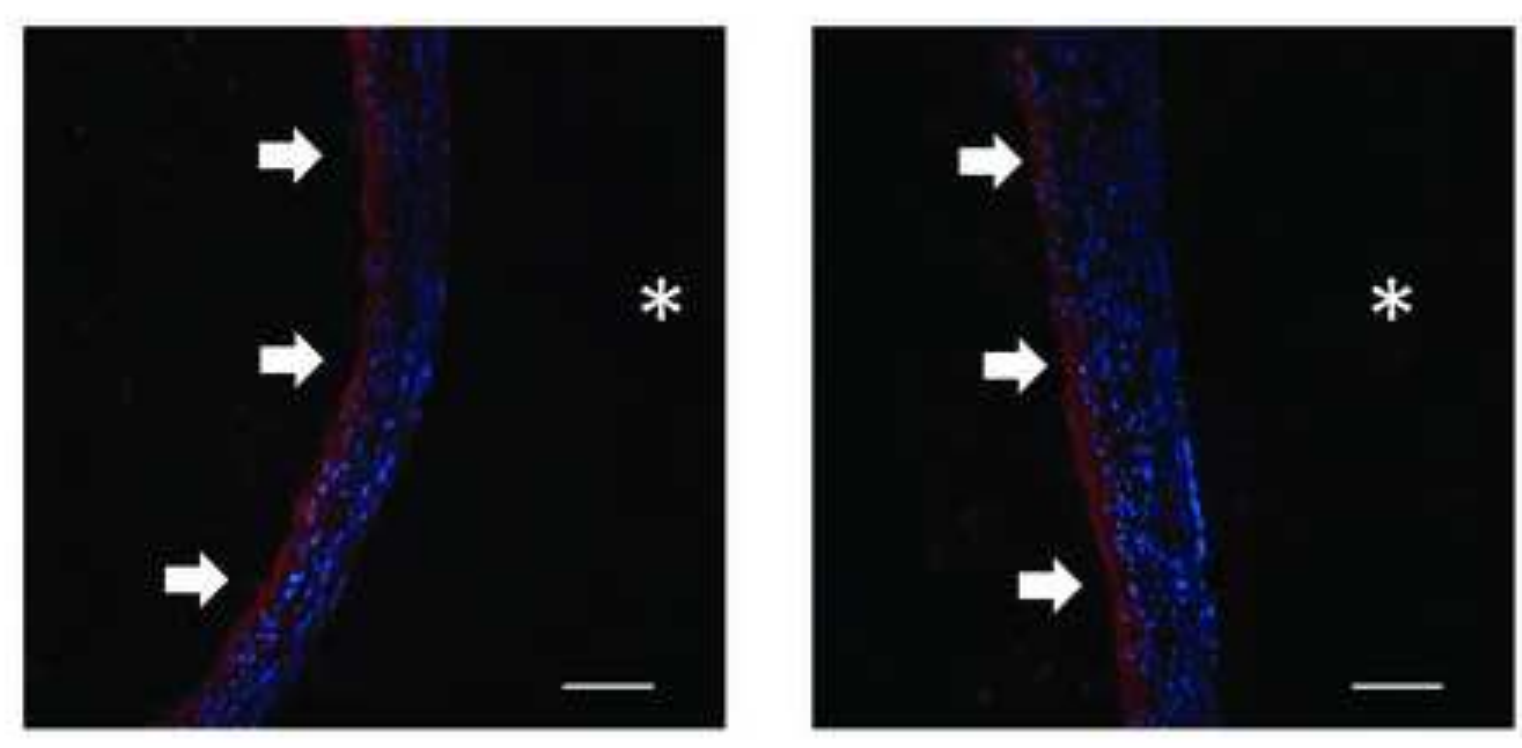

*

* 


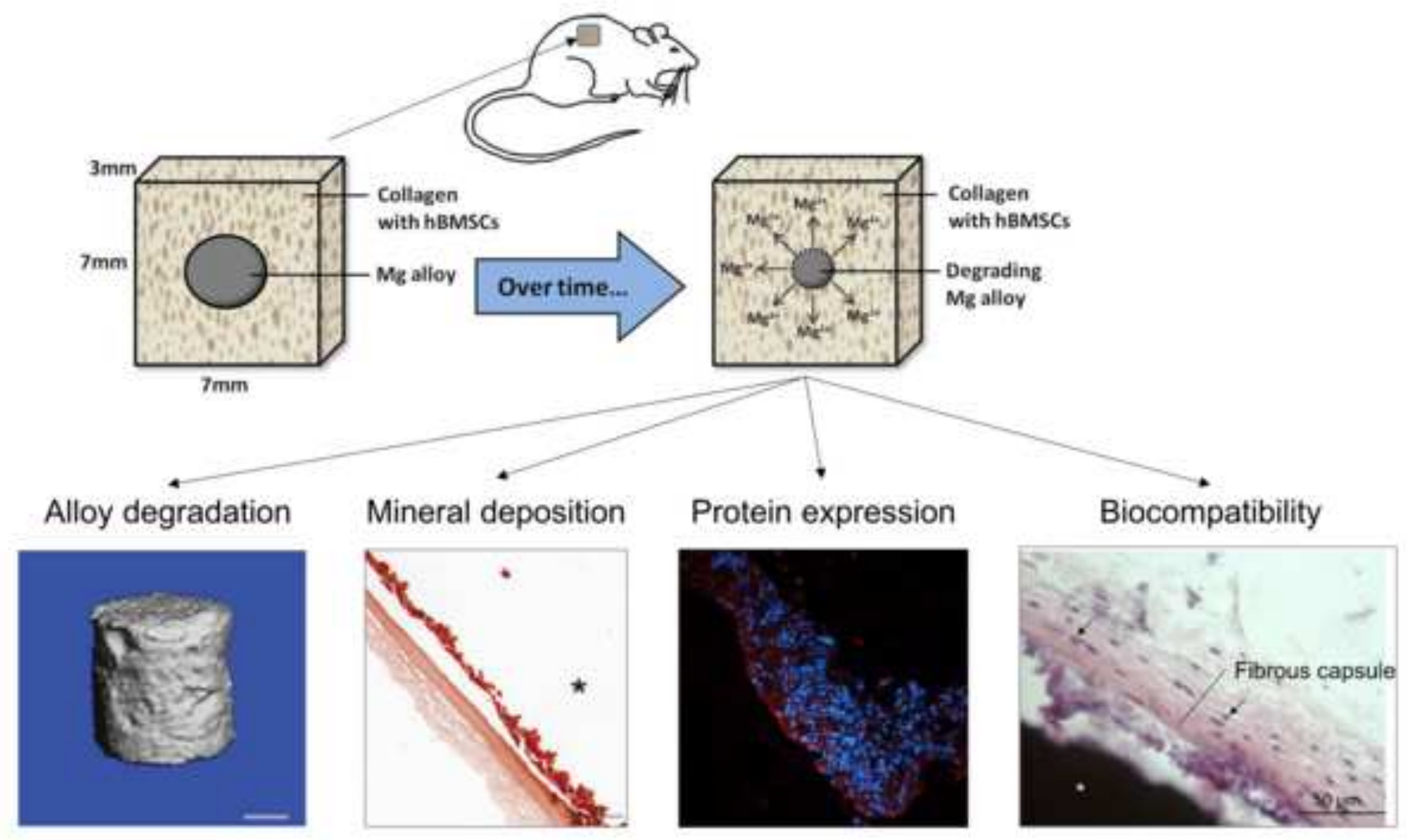

